\title{
A 2-year point-prevalence surveillance of healthcare-associated infections and antimicrobial use in Ferrara University Hospital, Italy
}

\author{
Paola Antonioli ${ }^{1} \mathbb{B}$, Niccolò Bolognesi ${ }^{2} \mathbb{B}$, Giorgia Valpiani ${ }^{3} \mathbb{D}$, Chiara Morotti ${ }^{3} \mathbb{D}$, Daniele Bernardini ${ }^{2} \mathbb{D}$, \\ Francesca Bravi ${ }^{3}$ (D) Eugenio Di Ruscio ${ }^{4}$ (D) Armando Stefanati ${ }^{5}$ (D) and Giovanni Gabutti $i^{5^{*}}$ (D)
}

\begin{abstract}
Background: Healthcare-Associated Infections (HAls) represent one of the leading issues to patient safety as well as a significant economic burden. Similarly, Antimicrobial Use (AMU) and Resistance (AMR) represent a growing threat to global public health and the sustainability of healthcare services.

Methods: A Point Prevalence Survey (PPS) following the 2016 ECDC protocol for HAl prevalence and AMU was conducted at Ferrara University Hospital (FUH). Data were collected by a team of trained independent surveyors in 2016 and 2018. Risk factors independently associated with HAI were assessed by a multivariate logistic regression model.

Results: Of the 1102 patients surveyed, 115 (10.4\%) had an active HAl and 487 (44.2\%) were on at least 1 systemic antimicrobial agent. Factors independently associated with increased HAl risk were a "Rapidly Fatal" McCabe score (expected fatal outcome within 1 year), presence of medical devices (PVC, CVC, indwelling urinary catheter or mechanically assisted ventilation) and a length of hospital stay of at least 1 week. The most frequent types of HAl were pneumonia, bloodstream infections, and urinary tract infections. Antimicrobial resistance to third-generation cephalosporins was observed in about $60 \%$ of Enterobacteriaceae.
\end{abstract}

Conclusions: The survey reports a high prevalence of HAI and AMU in FUH. Repeated PPSs are useful to control HAls and AMU in large acute-care hospitals, highlighting the main problematic factors and allowing planning for improvement actions.

Keywords: Infection control, Cross infection, latrogenic disease, Antimicrobial stewardship, Drug resistance, bacterial, Point prevalence survey

\section{Background}

Healthcare-associated infections (HAIs) represent one of the leading issues to patient safety as well as a significant economic burden on healthcare systems [1].

About 3.2 million HAIs occur every year in the European Union (EU) [2], causing 37,000 deaths as a direct consequence [3], over 2.5 million Disability Adjusted Life Years (DALYs) [4] and 16 million extra days of hospitalization, with

\footnotetext{
* Correspondence: giovanni.gabutti@unife.it

${ }^{5}$ Department of Medical Sciences, University of Ferrara, via Fossato di Mortara 64b, 44121 Ferrara, Italy

Full list of author information is available at the end of the article
}

an approximate cost of around 7 billion euros [3]. In 2016, the European Centre for Disease Prevention and Control (ECDC) estimated that the burden of six most frequent types of HAI (pneumonia, urinary tract infection - UTI, surgical site infection, Clostridium difficile infection) in the EU was higher than the combined impact of 31 other infectious diseases under ECDC surveillance [5].

In the USA, it has been estimated that the 5 most impacting HAIs have a cost of 9.8 billion dollars: surgical site infections, Ventilation-Associated Pneumonia (VAP), Central Venous Catheter (CVC) associated bloodstream infections,

(c) The Author(s). 2020 Open Access This article is distributed under the terms of the Creative Commons Attribution 4.0 International License (http://creativecommons.org/licenses/by/4.0/), which permits unrestricted use, distribution, and 
C. difficile infections, and UTIs accounting for 33.7, 31.6, $18.9,15.4$ and $<1 \%$ of total costs, respectively [6].

In Italy, about 450-700 thousand HAIs occur every year in hospitalized patients, of which $30 \%$ considered avoidable. Currently, an HAI monitoring system is still not available at national level, and related data are limited to prevalence studies [7].

Similarly, antimicrobial use (AMU) and resistance (AMR) represent a growing threat to global public health and the sustainability of healthcare services [8]. The spreading of carbapenemase-producing Enterobacteriaceae since 2010 is associated with high lethality in hospital settings [9] and a global alert has been raised by the World Health Organization (WHO) [10]. A systematic review of infections with antibiotic-resistant bacteria in the EU estimated about 670,000 cases in 2015, of which $63.5 \%$ were associated with healthcare. About 33, 000 deaths and 870,000 DALYs were attributable to these infections [11].

Italy is one of the European countries with the highest level of AMU and with the highest prevalence of AMR both in the community and in hospital settings $[12,13]$. In 2015, the project "Good practices for the surveillance and control of antimicrobial resistance" was funded by the Italian National Centre for Disease Prevention and Control $(\mathrm{CCM})$ in order to promote integrated actions at national level to control AMR [14].

In order to estimate the burden of HAIs and AMR in the EU, ECDC provided a standardized protocol for HAI Point Prevalence Survey (PPS) to allow data gathering and comparison. The first ECDC prevalence study in the EU was realized in 2011-2012 [2]. A second ECDC study (PPS2) has been conducted in 2016-2017 [13]. In Italy, HAI prevalence in PPS2 (2016) resulted equal to $8.0 \%[13,15]$. The most common infections were pneumonia (20.3\%), bloodstream infections (18.3), UTI (18\%), surgical site infections (14.4\%) and gastrointestinal infections $(8.5 \%)$.

In Ferrara University Hospital (FUH), HAI and AMR monitoring have begun in 1992. In $2011 \mathrm{FUH}$ has joined ECDC PPS. An internal survey was repeated with the same protocol in 2012 and 2013 and data were published [16]. Subsequently, FUH also participated in 2016-2017 ECDC PPS. Additionally, FUH implemented for a long time risk management and infection control actions, including surveillance of surgical site infections, UTI and bloodstream infections, antimicrobial stewardship [17] and an AMR alert system, retraining of healthcare workers and application of WHO Guidelines on hands hygiene [18]. In 2018 a Lean Healthcare Management program for infection control (Lean4Health) was adopted in FUH, establishing improvement actions in Rehabilitation and Surgical departments [19].
The objective of this study was to determine the (1) prevalence of HAI and associated risk factors, (2) distribution of systemic AMU and AMR trend in FUH, comparing prevalence data recorded in 2016 and 2018.

\section{Methods}

The point prevalence survey took place from November 14th through 30th of 2016 and from November 12th through 23rd of 2018 in FUH, a tertiary care hospital in Northern Italy accounting 658 beds (578 acute-care, 70 rehabilitation, 10 post-acute care).

Study protocol was approved by the Independent Ethical Committee of Area Vasta Emilia Centrale (CE-AVEC, study code: 638 t2018/Oss/AOUFe; date of approval CE: $17 / 10 / 2018)$. As no information which may identify the subjects was collected, no informed consent was obtained.

Both surveys followed the ECDC PPS protocol version 5.3 [20]. In order to eliminate potential sources of conflict of interest, under-reporting of HAIs and variability of results, data were gathered by trained, independent surveyors (resident doctors of the Postgraduate School of Hygiene and Preventive Medicine of University of Ferrara) and one survey leader (Infection Risk Manager of FUH), consulting clinical documentation and interviewing Medical and Assistant Referents for the management of infectious risk in each department.

All inpatients and newborns admitted to the wards before 8:00 AM and not discharged at the time of the survey were included as indicated by the ECDC protocol [20]. Patients admitted to the emergency department and day surgery were excluded. Data collection in each ward was completed within the same day.

Data included admission date, patient demographics, systemic antimicrobial therapy, active HAIs, surgical procedures (using National Health Safety Network NHSN categories [20]) and medical devices presence at the time of survey or on the HAI onset date (urinary catheter, CVC, peripheral venous catheter - PVC, mechanical ventilation), antimicrobial resistance data when blood culture was available and patients underlying medical conditions through McCabe score [21]. McCabe score is a classification of the severity of patient's comorbidities, including chronic conditions and conditions impairing immunological system (for example diabetes requiring amputation or post amputation, end-stage hematological malignancies, chronic leukemias, metastatic carcinoma).

Active HAI definition required infection symptoms on the survey day or systemic antimicrobial treatment on the survey day for symptoms present previously and to meet ECDC surveillance criteria for HAI [20]. Antimicrobials have been categorized accordingly to the Anatomical Therapeutic Chemical classification (ATC) [22]. 
Time at risk is the time in days elapsed from the date of admission to the date of the survey. It has been categorized in 0 ( $0-6$ days) and 1 ( $>6$ days). The cutoff of 6 days has been chosen accordingly to the median time of hospital stay on survey date resulted in the Italian PPS2 Report 2016/2017 [15].

\section{Statistical analysis}

Categorical data were expressed as total numbers and percentages. The Shapiro-Wilk test was used to test for normality of the distribution of the continuous variables. In the presence of symmetry of the distributions, the variables will be represented with the mean and Standard Deviation (SD) or, in the case of non-normal distribution, with the median value and Interquartile Range [IQR - 1st quartile 3rd quartile]. Statistical comparisons of categorical variables were assessed using Pearson's $\chi 2$ test or Fisher's exact test depending on the minimal expected count in each crosstab. Unadjusted logistic regression analysis was performed to estimate Odds Ratios (ORs) and respective 95\% Confidence Intervals $(95 \% \mathrm{CIs})$. All variables were allowed entry in the multivariate logistic regression model, including those that resulted statistically not significant in the univariate analysis. The final multivariate logistic regression model has been estimated using a backward elimination stepwise procedure with a significance level of $p<0.05$ for a variable to stay in the model. Model calibration was assessed using the HosmerLemeshow goodness-of-fit test [23]. Area under the Receiver-Operating Curve (AROC) was used to assess discrimination power of the model.

Data was recorded with ECDC provided software HELICSWin.net v.1.3. All analyses were performed using STATA/SE ver 13.1 (Stata Corporation, College Station, Texas, USA). $P$-value $<0.05$ was defined as statistically significant.

\section{Results}

\section{Patient baseline characteristics}

A total of 1103 patients were included in the two surveys; 1 patient was excluded for incomplete data entry. Therefore, 1102 patients were included in the final analysis (530 in 2016, 572 in 2018). The demographics and clinical characteristics of patients are summarized in Table 1 . The median age was 71 [IQR 56 82] years and the median duration of hospital stay was 7 [IQR 3 15] days.

Of the patients surveyed, 337 (30.5\%) underwent at least one surgical procedure since admission, of which 170 (50.4\%) had major surgeries according to ECDC criteria.

\section{Factors associated with HAls}

In the unadjusted analysis patients with worst McCabe Scores (Ultimately Fatal and Rapidly Fatal disease), that had CVC, indwelling urinary catheter, mechanically assisted ventilation or hospitalized for more than 6 days since admission were at an increased risk of HAI. (Table 1).

Multivariate logistic regression showed that patients with "Rapidly Fatal" McCabe Scores, who had PVC, CVC, indwelling urinary catheter or mechanically assisted ventilation, or hospitalized for more than 6 days since admission were at an increased risk of HAI. (Table 1).

\section{Prevalence of HAls}

Overall HAI prevalence was $10.4 \%$ (patients with at least one HAI), 10.0\% in 2016 (53 patients) and 11.0\% in 2018 (63 patients). In acute-care wards HAI prevalence resulted 9.9\% in 2016 (9.2\% in medical departments, $7.6 \%$ surgical departments, $35.0 \%$ intensive care units) and $11.8 \%$ in 2018 (12.4\% in medical departments, $6.8 \%$ in surgical departments, $36.8 \%$ in intensive care units). In rehabilitation wards prevalence resulted $12.1 \%$ in 2016 and $5.8 \%$ in 2018. No HAIs were present in post-acute care.

Total count of HAIs resulted 63 in 2016 and 74 in 2018. Most common infection types in 2016 were: pneumonia (27.0\%), UTI (25.4\%), bloodstream infections (15.9\%), gastrointestinal infections (9.5\%), surgical site infections (6.3\%) and clinical sepsis (6.3\%). In 2018 were pneumonia (32.4\%), bloodstream infections (21.6\%), gastrointestinal infections (10.8\%), surgical site infections (9.5\%), UTI (6.8\%). 20162018 trends in HAIs resulted statistically significant for UTIs only $(p=0.003)$. See Table 2 for complete results.

\section{Antimicrobial use}

Four hundred eighty-four inpatients (44.2\%) were receiving at least one antimicrobial drug, 239 (45.0\%) in 2016 and 245 (42.8\%) in 2018, respectively. Total prescribed antimicrobial count resulted 348 in 2016 and 345 in 2018. ATC most frequent antimicrobial classes were: "combinations of penicillins, including beta-lactamase inhibitors" (27.3\% in 2016 and $23.8 \%$ in 2018), "thirdgeneration cephalosporins" (20.7\% in 2016 and $22.6 \%$ in 2018), "fluoroquinolones" (8.0\% in $2016,7.0 \%$ in 2018). 2016-2018 trends in AMU resulted not significant. (Table 2).

\section{HAl pathogens and antimicrobials resistance}

At the time of the surveys, microbiological data were available for 59 HAI (20 in 2016 and 39 in 2018). Most frequent pathogens resulted $C$. difficile (16.9\%), K. pneumoniae (11.9\%), C. albicans (8.5\%), E. coli (8.5\%) and S. epidermidis (8.5\%), S. aureus (6.8\%), S. maltophilia (6.8\%). Of these, $60.0 \%$ of $E$. coli were nonsusceptible to third-generation cephalosporins. Nonsusceptibility to third generation cephalosporins and carbapenems was present in 57.1 and $14.2 \%$ of $K$. pneumoniae, respectively. S. aureus was Oxacillin resistant in 1 case (25.0\%). 
Table 1 Baseline Characteristics and Risk Factors for Healthcare-Associated Infections

\begin{tabular}{|c|c|c|c|c|c|c|c|c|c|}
\hline \multirow[b]{2}{*}{ Patient Characteristics } & \multirow[b]{2}{*}{ Patients without HAl } & \multirow[b]{2}{*}{ Patientswith HAl } & \multicolumn{3}{|c|}{ Unadjusted logistic regression } & \multicolumn{4}{|c|}{ Adjusted logistic regression } \\
\hline & & & & $95 \%$ & $\mathrm{Cl}$ & & $95 \%$ & $\mathrm{Cl}$ & \\
\hline & $n=987$ & $n=115$ & OR & lower & upper & OR & lower & upper & $p$-value \\
\hline \multicolumn{10}{|l|}{ Sex } \\
\hline female, $n(\%)$ & $498(50.5)$ & $57(49.5)$ & 1.04 & 0.70 & 1.52 & & & & \\
\hline \multicolumn{10}{|l|}{ Age, years } \\
\hline median [IQR] & $71[5682]$ & 71 [57 82] & 1.01 & 0.99 & 1.01 & & & & \\
\hline \multicolumn{10}{|l|}{ Age classes, $n(\%)$} \\
\hline $15-64$ years & $345(35.0)$ & 39 (33.9) & Ref & & & & & & \\
\hline $0-1$ years & $29(2.9)$ & $3(2.6)$ & 0.92 & 0.27 & 3.14 & & & & \\
\hline $2-14$ years & $15(1.5)$ & $0(0.0)$ & - & - & - & & & & \\
\hline$>64$ years & $598(60.6)$ & $73(63.5)$ & 1.08 & 0.72 & 1.63 & & & & \\
\hline \multicolumn{10}{|l|}{ McCabe Score, $n$ (\%) } \\
\hline Non-fatal disease & $594(61.0)$ & $30(27.0)$ & Ref & & & & & & \\
\hline Ultimately fatal disease & $233(24.0)$ & $35(31.5)$ & 2.97 & 1.78 & 4.96 & & & & \\
\hline Rapidly fatal disease & $146(15.0)$ & $46(41.5)$ & 6.23 & 3.80 & 10.22 & 2.63 & 1.59 & 4.36 & 0.001 \\
\hline \multicolumn{10}{|l|}{ Medical Devices, $n$ (\%) } \\
\hline Presence of PVC & $674(68.3)$ & $81(71.7)$ & 1.18 & 0.76 & 1.81 & 2.80 & 1.57 & 4.99 & 0.001 \\
\hline Presence of urinary catheter & $346(35.2)$ & $73(64.6)$ & 3.36 & 2.24 & 5.05 & 1.78 & 1.10 & 2.90 & 0.019 \\
\hline Presence of CVC & $120(12.2)$ & $52(45.2)$ & 5.96 & 3.94 & 9.02 & 3.59 & 1.98 & 6.51 & $<0.001$ \\
\hline Presence of MV & $18(1.8)$ & $17(14.9)$ & 9.43 & 4.71 & 18.90 & 4.13 & 1.68 & 10.19 & 0.002 \\
\hline \multicolumn{10}{|l|}{ Surgery, n (\%) } \\
\hline None & $688(69.9)$ & $75(65.2)$ & Ref & & & & & & \\
\hline Non-NHSN & $148(15.0)$ & $19(16.5)$ & 1.18 & 0.69 & 2.01 & & & & \\
\hline NHSN & $149(15.1)$ & $21(18.3)$ & 1.29 & 0.77 & 2.16 & & & & \\
\hline \multicolumn{10}{|l|}{ Time at risk*, days } \\
\hline median [IQR] & $6\left[\begin{array}{ll}2 & 13\end{array}\right]$ & 15 [9 29] & 1.01 & 1.003 & 1.011 & & & & \\
\hline \multicolumn{10}{|l|}{ Time at risk, $n(\%)$} \\
\hline$>6$ days & $472(47.8)$ & $103(89.6)$ & 9.37 & 5.08 & 17.24 & 9.38 & 4.87 & 18.08 & $<0.001$ \\
\hline \multicolumn{10}{|l|}{ Year of the survey, $n$ (\%) } \\
\hline 2016 & $478(48.4)$ & $52(45.2)$ & Ref & & & & & & \\
\hline 2018 & $509(51.6)$ & $63(54.8)$ & 1.14 & 0.77 & 1.68 & & & & \\
\hline
\end{tabular}

Abbreviations: HAI Healthcare-Associated Infection, IQR Interquartile Range, PVC Peripheral Vascular Catheter, CVC Central Venous Catheter, MV Mechanical Ventilation, NHSN National Health Safety Network, OR Odds Ratio, Cl Confidence Interval, Ref Reference Category

*Time elapsed from date of admission to date of survey

Model log-likelihood $=-265.84 ; \mathrm{LR} X 2=170.8, p<0.001$. Hosmer-Lemeshow $\mathrm{X} 2=6.81, p=0.4486$. Area under receiver operating characteristics curve (AROC) $=$ 0.84. Values in bold are statistically significant $(p<0.05)$

\section{Discussion}

The main results of this study were that $10.4 \%$ of inpatients had at least $1 \mathrm{HAI}$ and $44.2 \%$ inpatients was on at least 1 systemic antimicrobial agent, without any statistically significant difference observed between 2016 and 2018 surveys. AMU shows a constantly decreasing trend in FUH surveys $(54.4 \%$ in $2011,50.1 \%$ in $2012,48.4 \%$ in $2013,45.0 \%$ in $2016,42.8 \%$ in 2018,16$]$.

The prevalence of HAIs observed in FUH resulted higher than in other studies conducted in the EU and based on ECDC protocol. EU corrected HAI prevalence after validation for PPS2 resulted $6.5 \%$ in 2016. In this study, reported HAI prevalence for Italy over a sample of 14,476 patients was $8.0 \%$, one of the highest values in the EU countries [13]. However, in PPS2 HAI prevalence was higher in larger hospital accounting more than 500 beds $(9.32 \%)$ and lower in smaller hospitals (5.97\%). The proportion of patients undergoing at least 1 systemic antimicrobial agent was similar (44.5\%) [15]. A multicentric PPS study in Switzerland observed an HAI prevalence of 5.6\% [24]. The first national PPS in Singapore acute-care hospitals reported similar results for HAI prevalence (11.9\%) and a higher AMU (51.0\%) [25]. 
Table 2 Prevalence, clinical setting, infection site of Healthcare-Associated Infections and Antimicrobial Use by year of survey

\begin{tabular}{|c|c|c|c|}
\hline & 2016 & 2018 & Total \\
\hline Total No. of Patients, $n$ (\%) & $530(48.1)$ & $572(51.9)$ & $1102(100.0)$ \\
\hline HAI Prevalence (at least one), $n$ (\%)† & $52(9.8)$ & $63(11.0)$ & $115(10.4)$ \\
\hline Antimicrobials use prevalence (at least one), $n(\%) \dagger$ & $239(45.0)$ & $248(42.8)$ & $487(44.2)$ \\
\hline HAl by clinical setting, $n(\%)^{*}+$ & 52 & 63 & 115 \\
\hline Surgery & $9(17.3)$ & $6(9.5)$ & $15(13.0)$ \\
\hline Medicine & $27(51.9)$ & $40(63.5)$ & $67(58.3)$ \\
\hline Geriatrics & $0(0.0)$ & $1(1.6)$ & $1(0.9)$ \\
\hline Intensive Care & $7(13.5)$ & $7(11.1)$ & $14(12.2)$ \\
\hline Gynaecology/Obstetrics & $0(0.0)$ & $3(4.8)$ & $3(2.6)$ \\
\hline Paediatrics/Neonatology & $1(1.9)$ & $2(3.2)$ & $3(2.6)$ \\
\hline Rehabilitation & $8(1.5)$ & $4(6.3)$ & $12(10.4)$ \\
\hline Post-acute care & $0(0.0)$ & $0(0.0)$ & $0(0.0)$ \\
\hline Total No. of HAl & 63 & 74 & 137 \\
\hline \multicolumn{4}{|l|}{ HAl by infection site, $n(\%) \neq$} \\
\hline Pneumonia & $17(27.0)$ & $24(32.4)$ & $41(29.9)$ \\
\hline Bloodstream Infections & $10(15.9)$ & $16(21.6)$ & $26(19.0)$ \\
\hline Urinary Tract Infectionsł & $16(25.4)$ & $5(6.8)$ & $21(15.4)$ \\
\hline Gastro-intestinal system infections & $6(9.5)$ & $8(10.8)$ & $14(10.2)$ \\
\hline Surgical site infections & $4(6.3)$ & $7(9.5)$ & $11(8.0)$ \\
\hline Clinical sepsis & $4(6.3)$ & $4(5.4)$ & $8(5.8)$ \\
\hline Infections of ear, nose, throat, larynx and mouth & $1(1.6)$ & $5(6.8)$ & $6(4.4)$ \\
\hline Cellulitis, wound, deep soft tissue not involving bone, not related to surgery & $0(0.0)$ & $4(5.4)$ & $4(2.9)$ \\
\hline Lower respiratory tract infections & $2(3.2)$ & $1(1.4)$ & $3(2.2)$ \\
\hline Others & $3(4.8)$ & $0(0.0)$ & $3(2.2)$ \\
\hline Total No. of antimicrobials & 348 & 345 & 693 \\
\hline \multicolumn{4}{|l|}{ ATC Antimicrobial classes, $n(\%) \dagger$} \\
\hline J01CR Combinations of penicillins, incl. Beta-lactamase inhibitors & $95(27.3)$ & $82(23.8)$ & $177(25.6)$ \\
\hline J01DD Third-generation cephalosporins & $72(20.7)$ & $78(22.6)$ & $150(21.6)$ \\
\hline J01MA Fluorochinolones & $28(8.0)$ & $24(7.0)$ & $52(7.5)$ \\
\hline J01FA Macrolides & $13(3.7)$ & $20(5.8)$ & $33(4.7)$ \\
\hline J02 AC Triazole derivatives & $15(4.3)$ & $17(4.9)$ & $32(4.6)$ \\
\hline J01DH Carbapenems & $16(4.6)$ & $15(4.3)$ & $31(4.5)$ \\
\hline J01XD Imidazole derivatives & $17(4.9)$ & $14(4.1)$ & $31(4.5)$ \\
\hline J01XX08 Linezolid & $13(3.7)$ & $16(4.6)$ & $29(4.1)$ \\
\hline J01XA Glycopeptide antibacterials & $12(3.4)$ & $15(4.3)$ & $27(3.8)$ \\
\hline J01DB First-generation cephalosporins & $11(3.2)$ & $14(4.1)$ & $25(3.6)$ \\
\hline J01FF Lincosamides & $9(2.6)$ & $8(2.3)$ & $17(2.5)$ \\
\hline J01CA Penicillins with extended spectrum & $8(2.3)$ & $7(2.0)$ & $15(2.2)$ \\
\hline J01GB Aminoglycosides & $12(3.4)$ & $2(0.6)$ & $14(2.0)$ \\
\hline A07AA Intestinal anti-infectives antibiotics & $0(0.0)$ & $8(2.3)$ & $8(1.1)$ \\
\hline J01XX09 Daptomycin & $3(0.9)$ & $4(1.2)$ & $7(1.0)$ \\
\hline Others & $24(6.9)$ & $21(6.1)$ & $45(6.5)$ \\
\hline
\end{tabular}

Abbreviations: HAI Healthcare-Associated Infection, AMU Antimicrobial Use, ATC Anatomical Therapeutic Chemical Classification. *Percentages have been calculated on HAI prevalence in 2016 and 2018, respectively

$\dagger p$-value $=$ not significant; $\neq p$-value $<0.05$ 
As in other studies, intensive care unit was the clinical setting with higher HAI prevalence (35.0\% in 2016 and $36.8 \%$ in 2018, 2,15,16,24].

Among all inpatients, factors independently associated with increased HAI risk were a presence of medical devices (PVC, CVC, indwelling urinary catheter or mechanically assisted ventilation) a length of hospital stay of at least 1 week and a "Rapidly Fatal" McCabe score (expected fatal outcome within 1 year).

Regarding the type of HAI, pneumonia, bloodstream infections and UTI were the three most frequent considering both surveys. However, UTI significantly decreased in 2018 (from 25.4 to 6.5\%), dropping behind gastrointestinal system infections and surgical site infections. This could be explained by the Lean Healthcare Management program that was carried out in FUH in 2018, establishing improvement actions in Rehabilitation and Surgical departments and the update of FUH standard operating procedure for prevention of catheter-associated urinary tract infection according to the latest available evidence-based guidelines [26-29]. In 2019, the Lean Healthcare Management program has been extended to prevention and management of bloodstream infections in all hospital wards, including the Emergency Department.

The most frequent causative HAI pathogen was $C$. difficile (CD). Infections caused by $\mathrm{CD}$ represent one of the 5 most financially impacting HAIs [6]. American College of Gastroenterology "Guidelines for diagnosis, treatment and prevention of $\mathrm{CD}$ infections" report that antibiotics are the main risk factor for $\mathrm{CD}$ infections, particularly cephalosporin, fluoroquinolones and clindamycin [30]. These three drugs classes represent $30 \%$ of all systemic antimicrobials prescribed in FUH. Studies confirm the importance of antimicrobial stewardship, pointing out a reduction in $\mathrm{CD}$ infections incidence up to $60 \%$ [31-33]. Furthermore, AMR to third-generation cephalosporins (the second most frequently prescribed systemic antimicrobial in FUH) was observed in about $60 \%$ of Enterobacteriaceae and this family of microorganism was the causative pathogen of $25 \%$ of all HAI.

\section{Limitations}

These surveys have some limitations. First, our study considers a large acute-care hospital, therefore results are not generalizable for smaller hospitals $(<500$ patients). As pointed out in the second Italian PPS, HAI prevalence may indeed vary greatly with the hospital number of beds and the case-mix [15]. Second, data on AMR are limited because only a small proportion of microorganisms were tested as part of diagnosing.

\section{Conclusions}

Results of this study contribute to reinforce the statement that HAI and AMR remain a high burden for healthcare systems, undermining patient safety in hospitals and causing high rates of morbidity, mortality and costs [6].

Twenty years since the report To Err is Human [34], hospitals still need to be made safer, adopting evidencebased protocols for medical devices management and strict application of infection control guidelines, especially for frail patients with short life expectancy and prolonged hospital stays, in order to prevent HAI, reduce AMU and limit AMR.

PPSs represent a fast, easily repeatable, and not expensive method to accomplish HAI and AMU surveillance in health-care structures, pointing out priority areas that need improvement actions and providing feedback to health care professionals. Furthermore, the ECDC PPS protocol has been used worldwide [25, 35], allowing comparisons among different studies.

\section{Abbreviations}

AMR: Antimicrobial Resistance; AMU: Antimicrobial Use; AROC: Area under the Receiver-Operating Curve; ATC: Anatomical Therapeutic Chemical classification; CCM: Italian National Centre for Disease Prevention and Control; Cl: Confidence Intervals; CVC: Central Venous Catheter;

DALY: Disability Adjusted Life Years; ECDC: European Centre for Disease Prevention and Control; EU: European Union; FUH: Ferrara University Hospital; HAl: Healthcare Associated Infections; IQR: Interquartile Range; MV: Mechanical Ventilation; NHSN: National Health Safety Network; OR: Odds Ratio; PPS: Point Prevalence Survey; PVC: Peripheral Venous Catheter; Ref: Reference Category; UTI: Urinary Tract Infections; VAP: Ventilation Associated Prenumonia; WHO: World Health Organization

\section{Acknowledgments}

We thank FUH infection control referents for their collaboration and resident doctors of Postgraduate School of Preventive Medicine and Hygiene of the University of Ferrara for data collection (Umberto Cairoli, Gianluca Marini, Andrea Rauzino, Caterina Florescu, Federica Sandri, Cecilia Martellucci, Diego Gamberoni, Lucia Bertoni, Matteo Pellegrini).

\section{Authors' contributions}

PA conceived, designed and revised the work. NB contributed to acquisition, analysis, interpretation of data and was a major contributor in writing the manuscript. GV, CM contributed substantively to the analysis of data. DB contributed to acquisition of data. FB, EDR, AS, GG substantively revised the work. All authors read and approved the final manuscript.

Funding

No funding.

Availability of data and materials

The datasets used and analyzed during the current study are available from the corresponding author on reasonable request.

\section{Ethics approval and consent to participate}

Study protocol was approved by the Independent Ethical Committee of Area Vasta Emilia Centrale (CE-AVEC, study code: 638 t2018/Oss/AOUFe; date of approval CE: 17/10/2018). Data gathering was conducted following the principles of the Declaration of Helsinki, according to current national legislation and in compliance with the protection of personal data. As no information which may identify the subjects was collected, no informed consent was obtained.

Consent for publication

Not applicable. 


\section{Competing interests}

PA reports collaboration with Assobiomedica taking part in a Lean Management program for infection control (Lean4Health) without getting any grant.

GG reports, outside this manuscript, grants from Sanofi Pasteur MSD, GSK Biologicals SA, Novartis, Pfizer, Sanofi Pasteur, MSD Italy, PaxVax and Seqirus for taking part to advisory boards, expert meetings, for acting as speaker and/or organizer of meetings/congresses and as principal investigator and chief of O.U. in RCTs.

$\mathrm{NB}, \mathrm{GV}, \mathrm{CM}, \mathrm{DM}, \mathrm{FB}, \mathrm{EDR}$, AS declare no competing interests.

\section{Author details}

${ }^{1}$ Department of Hospital Hygiene \& Healthcare-Associated Infection Risk Management, Hospital Health Medical Management, S. Anna University Hospital of Ferrara, Ferrara, Italy. ${ }^{2}$ Postgraduate School of Hygiene and Preventive Medicine, University of Ferrara, Ferrara, Italy. ${ }^{3}$ Research Innovation Quality and Accreditation Unit, S. Anna University Hospital of Ferrara, Ferrara, Italy. ${ }^{4}$ Medical Director, S. Anna University Hospital of Ferrara, Ferrara, Italy. ${ }^{5}$ Department of Medical Sciences, University of Ferrara, via Fossato di Mortara 64b, 44121 Ferrara, Italy.

\section{Received: 11 September 2019 Accepted: 14 January 2020} Published online: 23 January 2020

\section{References}

1. Agency for Healthcare Research and Quality (U.S. Department of Health \& Human Services). Health Care-Associated Infections 2018. https://www.ahrq. gov/professionals/quality-patient-safety/patient-safety-resources/resources/ hais/index.html (accessed 22 January 2019).

2. European Centre for Disease Prevention and Control. Point prevalence survey of healthcare-associated infections and antimicrobial use in European acute care hospitals. Stockholm: ECDC: 2013. doi:https://doi.org/ $10.2900 / 86011$.

3. European Centre for Disease Prevention and Control. Annual Epidemiological Report 2012. Reporting on 2010 surveillance data and 2011 epidemic intelligence data. Stockholm: ECDC: 2013.

4. Cassini A, Plachouras D, Eckmanns T, Abu Sin M, Blank H-P, Ducomble T, et al. Burden of six healthcare-associated infections on European population health: estimating incidence-based disability-adjusted life years through a population prevalence-based Modelling study. PLoS Med. 2016;13:e1002150. https://doi.org/10.1371/journal.pmed.1002150.

5. Cassini A, Colzani E, Pini A, Mangen MJJ, Plass D, McDonald SA, et al. Impact of infectious diseases on population health using incidence-based disabilityadjusted life years (DALYs): results from the burden of communicable diseases in Europe study, European Union and European economic countries, 2009 to 2013. Eurosurveillance. 2018;23:1-20. https://doi.org/10. 2807/1560-7917.ES.2018.23.16.17-00454.

6. Zimlichman E, Henderson D, Tamir O, Franz C, Song P, Yamin CK, et al. Health care-associated infections. JAMA Intern Med. 2013;173:2039. https:// doi.org/10.1001/jamainternmed.2013.9763.

7. Istituto Superiore di Sanita. Infezioni correlate all'assistenza aspetti epidemiologici [Istituto Superiore di Sanità - Healthcare Associated Infections, epidemiological aspects] https://www.epicentro.iss.it/infezionicorrelate/epidemiologia (accessed 7 March 2019). Italian.

8. WHO. Antimicrobial resistance 2018. https://www.who.int/news-room/factsheets/detail/antimicrobial-resistance (accessed 22 January 2019).

9. Ramos-Castañeda JA, Ruano-Ravina A, Barbosa-Lorenzo R, PaillierGonzalez JE, Saldaña-Campos JC, Salinas DF, et al. Mortality due to KPC carbapenemase-producing Klebsiella pneumoniae infections: systematic review and meta-analysis: mortality due to KPC Klebsiella pneumoniae infections. J Inf Secur. 2018;76:438-48. https://doi.org/10.1016/j.jinf.2018. 02.007 .

10. Guidelines for the prevention and control of carbapenem-resistant Enterobacteriaceae, Acinetobacter baumannii and Pseudomonas aeruginosa in health care facilities. Geneva: World Health Organization; 2017. Licence: CC BY-NC-SA 3.0 IGO. https://apps.who.int/iris/bitstream/handle/10665/25 9462/9789241550178-eng.pdf;jsessionid=F0D861E42F91D8D48293FE5D3 OF99D75? sequence $=1$ (accessed 25 September 2019).

11. Cassini A, Högberg LD, Plachouras D, Quattrocchi A, Hoxha A, Simonsen GS, et al. Attributable deaths and disability-adjusted life-years caused by infections with antibiotic-resistant bacteria in the EU and the European economic area in 2015: a population-level modelling analysis. Lancet Infect Dis. 2019;19:56-66. https://doi.org/10.1016/\$1473-3099(18)30605-4.

12. European Centre for Disease Prevention and Control. Antimicrobial consumption. In: ECDC. Annual epidemiological report for 2017. Stockholm: ECDC; 2018. http://ecdc.europa.eu/sites/portal/files/documents/AER_for_201 7-antimicrobial-consumption.pdf ().

13. Suetens C, Latour K, Kärki T, Ricchizzi E, Kinross P, Moro ML, et al. Prevalence of healthcare-associated infections, estimated incidence and composite antimicrobial resistance index in acute care hospitals and long-term care facilities: results from two European point prevalence surveys, 2016 to 2017. Euro Surveill. 2018;23:15-28. https://doi.org/10.2807/1560-7917.ES.2018.23.46. 1800516.

14. Moro ML, Ciofi Degli Atti M, D’Amore C, Diegoli G, Forni S, Gagliotti C, et al. Good practices for the surveillance and control of antimicrobial resistance. Epidemiol Prev. 2019;43:185-93. https://doi.org/10.19191/EP19.2-3.P185.058.

15. Secondo studio di prevalenza italiano sulle infezioni correlate all'assistenza e sull'uso di antibiotici negli ospedali per acuti - Protocollo ECDC. Dipartimento Scienze della Salute Pubblica e Pediatriche Università di Torino. 2018. http://www.salute.gov.it/imgs/C_17_pubblicazioni_2791_ allegato.pdf (accessed 25 September 2019).

16. Antonioli P, Manzalini MC, Stefanati A, Bonato B, Verzola A, Formaglio A,Gabutti $G$. Temporal trends of healthcare associated infections and antimicrobial use in 2011-2013, observed with annual point prevalence surveys in Ferrara University hospital, Italy J Prev Med Hyg 2016;57(3):E135-E141. Review. PubMed PMID: 27980377; PubMed Central PMCID: PMC5139608.

17. Antonioli P, Formaglio A, Gamberoni D, Bertoni L, Perrone P, Stefanati A, et al. Hospital discharge in patients at risk of surgical site infection: antimicrobial stewardship at Ferrara University hospital, Italy. J Prev Med Hyg. 2018;59:E139-44.

18. WHO. WHO Guidelines on Hand Hygiene in Health Care 2009. https://www. who.int/gpsc/5may/tools/9789241597906/en/ (accessed 6 March 2019).

19. Antonioli P, Manzalini M, Bolognesi N, Martellucci C, Cudini E, Di Ruscio E, et al. Aim to improve safety and empowerment in surgery: what to do to get ready for the best. Clin Heal Promot. 2019;vol. 9(Warsaw):18 https:// www.hphconferences.org/fileadmin/user_upload/conferences/Warsaw2019/ Abstractbook_2019_Warsaw.pdf / (

20. ECDC. Point prevalence survey of healthcare-associated infections and antimicrobial use in European acute care hospitals - protocol version 5.3. Stockholm: ECDC: 2016. http://ecdc.europa.eu/sites/portal/files/documents/ PPS-HAI-AMR-protocol.pdf (accessed 25 September 2019).

21. McCabe WR, Jackson GG. Gram-negative bacteremia. Arch Intern Med. 1962; 110:847. https://doi.org/10.1001/archinte.1962.03620240029006.

22. WHO Collaborating Centre for Drug Statistic Methodology. ATC/DDD Index 2019 2019. https://www.whocc.no/atc_ddd_index/ (accessed 30 January 2019).

23. Hosmer DW, Lemeshow S. Applied logistic regression. Hoboken, NJ, USA: John Wiley \& Sons, Inc.; 2000. https://doi.org/10.1002/0471722146.

24. Metsini A, Vazquez M, Sommerstein R, Marschall J, Voide C, Troillet $N$, Gardiol C, Pittet D, Zingg W, The Swissnoso network. Point prevalence of healthcare-associated infections and antibiotic use in three large Swiss acute-care hospitals Swiss Med Wkly 2018;148:w14617. doi: https://doi.org/ 10.4414/smw.2018.14617. eCollection 2018. PubMed PMID: 29698542.

25. Cai Y, Venkatachalam I, Tee NW, Tan TY, Kurup A, Wong SY, et al. Prevalence of healthcare-associated infections and antimicrobial use among adult inpatients in Singapore acute-care hospitals: results from the first National Point Prevalence Survey. Clin Infect Dis. 2017;64:S61-7. https://doi.org/10. 1093/cid/cix103.

26. Loveday HP, Wilson JA, Pratt RJ, Golsorkhi M, Tingle A, Bak A, et al. Epic3: national evidence-based guidelines for preventing healthcare-associated infections in NHS hospitals in England. J Hosp Infect. 2014;86(Suppl 1):S170. https://doi.org/10.1016/S0195-6701(13)60012-2.

27. Hooton TM, Bradley SF, Cardenas DD, Colgan R, Geerlings SE, Rice JC, et al. Diagnosis, prevention, and treatment of catheter-associated urinary tract infection in adults: 2009 international clinical practice guidelines from the Infectious Diseases Society of America. Clin Infect Dis. 2010;50:625-63. https://doi.org/10.1086/650482.

28. Tenke P, Kovacs B, Bjerklund Johansen TE, Matsumoto T, Tambyah PA, Naber KG. European and Asian guidelines on management and prevention of catheter-associated urinary tract infections. Int J Antimicrob Agents. 2008; 31:68-78. https://doi.org/10.1016/j.jjantimicag.2007.07.033.

29. Lo E, Nicolle LE, Coffin SE, Gould C, Maragakis LL, Meddings J, et al. Strategies to prevent catheter-associated urinary tract infections in acute 
care hospitals: 2014 update. Infect Control Hosp Epidemiol. 2014;35:464-79. https://doi.org/10.1086/675718.

30. Surawicz CM, Brandt $L$, Binion DG, Ananthakrishnan AN, Curry SR, Gilligan $\mathrm{PH}$, et al. Guidelines for diagnosis, treatment, and prevention of clostridium difficile infections. Am J Gastroenterol. 2013;108:478-98. https://doi.org/10. 1038/ajg.2013.4

31. Valiquette L, Cossette B, Garant M-P, Diab H, Pépin J. Impact of a reduction in the use of high-risk antibiotics on the course of an epidemic of Clostridium difficile-associated disease caused by the Hypervirulent NAP1/ 027 strain. Clin Infect Dis. 2007:45:S112-21. https://doi.org/10.1086/519258.

32. Climo MW, Israel DS, Wong ES, Williams D, Coudron P, Markowitz SM. Hospitalwide restriction of clindamycin: effect on the incidence of Clostridium difficileassociated diarrhea and cost. Ann Intern Med. 1998;128:989-95.

33. Wilcox MH, Freeman J, Fawley W, Mackinlay S, Brown A, Donaldson K, et al. Long-term surveillance of cefotaxime and piperacillin-tazobactam prescribing and incidence of Clostridium difficile diarrhoea. J Antimicrob Chemother. 2004;54:168-72. https://doi.org/10.1093/jac/dkh285.

34. Institute of Medicine (US) Committee on Quality of Health Care in America; Kohn LT, Corrigan JM, Donaldson MS, editors. To Err is Human: Building a Safer Health System. Washington (DC): National Academies Press (US); 2000. PubMed PMID: 25077248.

35. Saleem Z, Hassali MA, Godman B, Hashmi FK, Saleem F. A multicenter point prevalence survey of health care-associated infections in Pakistan: findings and implications. Am J Infect Control 2019:47(4):421-424. doi: https://doi. org/10.1016/j.ajic.2018.09.025. Epub 2018 Nov 22. PubMed PMID: 30471976

\section{Publisher's Note}

Springer Nature remains neutral with regard to jurisdictional claims in published maps and institutional affiliations.

Ready to submit your research? Choose BMC and benefit from:

- fast, convenient online submission

- thorough peer review by experienced researchers in your field

- rapid publication on acceptance

- support for research data, including large and complex data types

- gold Open Access which fosters wider collaboration and increased citations

- maximum visibility for your research: over $100 \mathrm{M}$ website views per year

At $\mathrm{BMC}$, research is always in progress.

Learn more biomedcentral.com/submissions 